\title{
Combined saphenous-sciatic nerve blockade superior to femoral-sciatic nerve blockade for postoperative analgesia following foot and ankle
}

\section{surgery}

\begin{abstract}
Introduction: Femoral nerve blockade combined with sciatic nerve blockade has been reported to be an effective treatment regimen for postoperative analgesia following lower extremity surgery.Saphenous nerve blockade is an alternative to femoral nerve blockade for postoperative analgesia in knee arthroplasty surgeryand the level and quality of analgesia that either technique provides patients have been reported to be equivalent. This study compared the postoperative analgesic properties of combined femoral and sciatic nerve blockade with those of combined saphenous and sciatic nerve blockade in patients that underwent foot and ankle surgery.
\end{abstract}

Method: All patients received general inhalational endotracheal anesthesia and were administered lateral popliteal sciatic nerve blockade by a combined nerve stimulator and ultrasound guidance technique in the postoperative recovery area. During the first six months of the study femoral nerve blockade was simultaneously administered employing combined nerve stimulation and ultrasound guidance. During the second six month interval saphenous nerve blockade was administered using ultrasound guidance. Continuous nerve block catheters were inserted if patients were admitted over 24 hours. Breakthrough pain was treated with hydro-morphone patient controlled analgesic infusions. Postoperative pain scores, opioid analgesic consumption and lower extremity movement in the immediate recovery period and at 24 hours after nerve blockade were recorded.

Results: 167 patients were enrolled. Femoral nerve blockade was administered to 45 patients and 122 received a saphenous nerve blockade. Immediate pain scores were lower for opioid naïve females that were administered saphenous nerve blockade. All groups that received saphenous nerve blockade had lower opioid requirements in the immediate postoperative period. Differences in pain scores and opioid requirements were noted between opioid naïve and tolerant patient groups.

Conclusion: Combined popliteal sciatic and saphenous nerve blockade resulted in lower immediate postoperative pain scores in opioid naïve females and reduced immediate postoperative rescue opioid analgesic dosages in all patient groups. Differences in pain scores and analgesic requirements were not present 24 hours postoperatively. Opioid tolerant patients with effective nerve blockade 24 hours postoperatively required elevated rescue opioid analgesic medication dosages.
Volume 3 Issue 3 - 2015

Loreto Lollo, Agnes Stogicza

Department of Anesthesiology \& Pain Medicine, USA

Correspondence: Lollo L, Department of Anesthesiology and Pain Medicine, University of Washington, 1959 NE Pacific St, BB-146, Seattle,WA 98195-6540, USA, Tel 206-543-2673, Fax 206-543-2958,Email lollomd@uw.edu

Received: October 07, 2015 | Published: October 29, 2015
Abbreviations: FNB, femoral nerve blockade; SNB, sciatic nerve blockade; SSNB, saphenous nerve blockade; BMI, body mass index; MS, morphine sulfate; PACU, post anesthesia care unit; PCA, patient controlled analgesia; ON, opioid naïve; OT, opioid tolerant

\section{Background}

Postoperative analgesia following foot and ankle surgery can be provided to patients by a variety of techniques, each with their own indications, advantages and limitations. ${ }^{1,2}$ Femoral nerve blockade (FNB) combined with sciatic nerve blockade (SNB) following total knee arthroplasty and foot and ankle surgery has been reported to be an effective treatment regimen for postoperative analgesia. ${ }^{3,4}$ Saphenous nerve blockade (SSNB) has several advantages as an alternative to FNB for postoperative analgesia in lower extremity surgery and these include the absence of quadriceps femoris weakness that promotes early patient ambulation, cost-effectiveness related to reduced hospital length of stay and reduced infectious risk for continuous nerve block catheters placed in the antero-medial thigh region in contrast to the groin crease. ${ }^{5-7}$ The mid-thigh trans-sartorial approach for blockade of the saphenous nerve has been demonstrated to have a high success rate for patient analgesia and ultrasound guided imaging has led to more common use of this technique. ${ }^{8-10}$ The level and quality of analgesia that either the FNB or the SSNB provides patients that have undergone knee surgery have been reported to be equivalent although these trials were undertaken with a nerve stimulator guided technique without the employment of ultrasound imaging. ${ }^{7,11}$ Proximal saphenous nerve blockade for foot and ankle surgery improves analgesia even in the absence of medial incisions or manipulations and is considered due to deep osseous innervation that is ineffectively anesthetized with local anesthetic infiltration at the level of the ankle. ${ }^{12}$ The purpose of this study was to compare the postoperative analgesic properties of combined femoral and sciatic nerve blockade (SF) with those of combined saphenous and sciatic nerve blockade (SS) in patients that underwent foot and ankle surgery. 


\section{Method}

After receiving institutional review board approval from the University of Washington Human Subjects Division, patients provided written informed consent prior to undergoing foot and ankle surgery and were enrolled for participation in this prospective study of the perioperative analgesic effects of popliteal sciatic and femoral or saphenous nerve blockade administered under general anesthesia. All procedures were performed at Harborview Medical Center, Seattle. The preoperative data collected were age, gender, ASA physical status, height, weight, calculated body mass index (BMI), recent traumatic lower extremity injury, pre-existing nerve deficits or neuropathy and chronic pain syndromes affecting the operative lower extremity, worst pain score in the preceding 24 hour interval and maintenance preoperative 24 hour opioid dosage converted to $\mathrm{mg}$ of intravenous morphine sulfate (MS).

All patients received general inhalational endotracheal anesthesia with sevoflurane and intraoperative analgesia in the form of intravenous fentanyl, morphine and/or hydromorphone for their surgery. The dose of intraoperative opioid administered was recorded for each patient. Postoperative popliteal sciatic nerve blockade (SNB) by the lateral approach at a point $10 \mathrm{~cm}$ proximal to the popliteal crease was performed in the post anesthesia care unit (PACU) by anesthesia resident physicians and nurse anesthetists supervised by regional anesthesia physician faculty with added expertise in ultrasound guided imaging. The level of training of the practitioner performing the SNB was recorded.

All patients were administered $25 \mathrm{ml} \quad 0.375 \% \quad(93.7 \mathrm{mg})$ bupivacaine for the SNB using a Life-Tech ProBloc II 20 Gauge $100 \mathrm{~mm} 30$ degree bevel needle. The procedure was performed with the use a Life-Tech Tracer III nerve stimulator (NS) combined with ultrasound (US) guidance using a SonoSite M Turbo with a linear $38 \mathrm{~mm}$ probe to locate the sciatic nerve in the short axis view proximal to its branch point using an in plane approach for needle visualization. The bolus dose of local anesthetic was injected when the needle tip was observed in close proximity to the nerve and nerve stimulation generated adequate toe plantar flexion observed at a current of less than $0.6 \mathrm{~mA}$ and greater than $0.3 \mathrm{~mA}$.

Continuous SNB was administered to patients that sustained extensive foot and ankle surgery and were admitted over 24 hours for postoperative care. A $20 \mathrm{Ga} 100 \mathrm{~mm}$ ProLong continuous nerve block needle was used to insert a 22 Ga nerve block catheter after injection of the local anesthetic bolus dose through the needle and its position was secured at a distance $3 \mathrm{~cm}$ beyond the needle tip. An infusion of $0.125 \%$ bupivacaine at $10 \mathrm{ml} / \mathrm{hr}(12.5 \mathrm{mg} / \mathrm{hr})$ was administered through the sciatic nerve catheter and this infusion was titrated upwards in increments of $2 \mathrm{ml} / \mathrm{hr}$ to a maximum rate of $14 \mathrm{ml} / \mathrm{hr}(17.5 \mathrm{mg} / \mathrm{hr})$ if the patient reported inadequate analgesia.

During the first six months of the study patients that sustained incisions and surgical interventions that included the medial side of the lower extremity also received either a single bolus or continuous femoral nerve block (FNB). The same equipment employed for SNB under nerve stimulator guidance was used to elicit quadriceps femor is or patellar tendon twitches at $0.3-0.6 \mathrm{~mA}$ intensity after the stimulating needle was inserted $2 \mathrm{~cm}$ lateral to the palpable femoral pulse in the inguinal region. FNB under ultrasound guidance used the same equipment listed for SNB and the technique employed short axis visualization with an in plane approach to place the needle in close proximity to the posterolateral aspect of the femoral artery in the inguinal region. After negative aspiration for blood a bolus dose of $12 \mathrm{~mL} 0.375 \%$ bupivacaine $(45 \mathrm{mg}$ ) were injected. Using the same equipment and local anesthetic infusions and rates for continuous $\mathrm{SNB}$, a continuous femoral nerve block catheter was inserted $3 \mathrm{~cm}$ distal to the needle tip if the hospital admission was greater than 24 hours.

During the second six month interval of this study, as an alternative to FNB, a single bolus or continuous saphenous nerve blockade (SSNB) was also used to provide analgesia when surgical procedures included the medial side of the lower extremity. SSNB was performed under ultrasound guidance using the same equipment listed for SNB and the technique employed short axis visualization with an in plane approach to place the needle in close proximity to the saphenous nerve in the subsartorial space at a point $10 \mathrm{~cm}$ proximal and medial to the medial femoral condyle. After negative aspiration for blood a bolus dose of $12 \mathrm{~mL} 0.375 \%$ bupivacaine ( $45 \mathrm{mg}$ ) were injected. Using the same equipment and infusions and rates for continuous SNB, a continuous saphenous nerve block catheter was inserted at a distance $3 \mathrm{~cm}$ beyond the needle tip if the hospital admission was greater than 24 hours.

Intravenous patient controlled analgesia (PCA) using hydromorphone was prescribed to all patients for relief of breakthrough pain at a rate of $0.2 \mathrm{mg}$ every 6 minutes with an escalation dose of $0.4 \mathrm{mg}$ without a 6 hour maximum dose lockout restriction. Postoperative analgesia in those patients with inadequate pain relief following nerve blockade was administered as intravenous fentanyl, morphine and/or hydromorphone in bolus doses and oral oxycodone. In order to quantify the opioids administered to patients in equivalent dosing units and to compare the opioid usage between patients as a result of the variety of analgesic narcotic medications administered peri-operatively due to both patient and prescribing practitioner preferences, all dosages were converted to equipotent values in $\mathrm{mg}$ of intravenous morphine sulphate (MS) using standardized opioid conversion formulae.

The patient maximum self-reported pain score, observation of voluntary toe plantar flexion, and the total postoperative opioid doses converted to $\mathrm{mg}$ of intravenous MS at the time of discharge from the post anesthesia care unit (PACU) and for the first 24 hour postoperative interval were recorded.

\section{Results}

167 patients were enrolled in this study. 45 patients were administered combined sciatic and femoral nerve blockade (SF) and 122 received combined sciatic and saphenous nerve blockade (SS). Table 1 summarizes the perioperative demographics for the patients enrolled in the study. Table 1 also lists the perioperative pain scores and opioid requirements for the patient categories enrolled in this study. All patients retained voluntary motor control of the lower extremity that had sustained nerve blockade.

\section{Discussion}

The patients were categorized as either opioid naïve $(\mathrm{ON})$ or opioid tolerant (OT). OT patients have been reported to be challenging with respect to perioperative pain control. ${ }^{13}$ Including both ON and OTpatients together in the SF and SS categories would have skewed the results toward higher pain scores and elevated opioid dosages in all observation periods and led to sampling errors given the small sample sizes in some of the groups. Figure 1 is a plot of the perioperative opioid doses consumed by the patients categorized by gender and prior opioid tolerance. 
Table I Perioperative demographics for patients receiving sciatic-femoral (SF) and sciatic-saphenous (SS) nerve blocks categorized by the patient being opioid naïve (ON) or opioid tolerant (OT). Opioid dosages are recorded as mg Morphine Sulphate

\begin{tabular}{|c|c|c|c|c|c|c|c|c|}
\hline Gender & Female & Female & Female & Female & Male & Male & Male & Male \\
\hline Opioid Naïve or Tolerant & ON & ON & OT & OT & ON & ON & OT & OT \\
\hline Nerve Block Technique & SF & SS & SF & SS & SF & SS & SF & SS \\
\hline Ultrasound Guided (\%) & 14.3 & 100 & 12.5 & 100 & 13.3 & 100 & 0 & 100 \\
\hline Number of Patients & 14 & 31 & 8 & 24 & 17 & 35 & 6 & 32 \\
\hline Age (years) & $54.6(13.3)$ & $58.1(14.2)$ & $47.5(14.6)$ & 48.1 (13.3) & $49.8(16.1)$ & $57.6(\mid 4.1)$ & $51.7(12.7)$ & $49(14.2)$ \\
\hline ASA Status & 2 & 2 & $2(1)$ & $2(1)$ & 2 & 2 & $2(1)$ & $2(1)$ \\
\hline BMI & $27.6(5.1)$ & $27.6(5)$ & 24.7 (3.7) & $30.4(8.6)$ & $26.8(3.9)$ & $31.5(11.3)$ & $27.6(4.6)$ & $28.7(6.2)$ \\
\hline Pre-existent Neuropathy & I & 7 & I & 8 & I & 9 & 1 & 10 \\
\hline Diabetes & 2 & 2 & 0 & 3 & 2 & 4 & 0 & 3 \\
\hline Preoperative Pain Score & $4(4)$ & $5(4)$ & $6(1)$ & $7(3)$ & $4(3)$ & $5(3)$ & $5(2)$ & $6(3)$ \\
\hline Preoperative Opioid Dose & 0 & 0 & $34(22.3)$ & $18.8(23.4)$ & 0 & 0 & $28.4(17.8)$ & $30.8(31.7)$ \\
\hline Intraoperative Opioid Dose & $29.2(11.9)$ & $24.4(13.5)$ & $47.9(22.4)$ & $34.2(22.8)$ & $39.6(17.8)$ & $28.4(12.4) *$ & $43.4(17.6)$ & $35.7(18.5)$ \\
\hline PACU Pain Score & $3(2)$ & $\mathrm{I}(2)^{\wedge}$ & $4(2)$ & $4(3)$ & $2(2)$ & $2(2)$ & $3(2)$ & $2(3)$ \\
\hline Total PACU Opioid Dose & $17.2(22.3)$ & $7.5(12.1)$ & $31.4(24.5)$ & $21.1(18.9)$ & $12.3(13.6)$ & $5.6(9.8) *$ & $22.3(28.9)$ & $9(13.9)$ \\
\hline 24 Hour Pain Score & $4(3)$ & $4(2)$ & $5(3)$ & $5(3)$ & $2(2)$ & $3(2)$ & $4(3)$ & $5(2)$ \\
\hline Total 24 Hour Opioid Dose & $59.3(31.8)$ & $46.2(5 I .7)$ & $150(98.8)$ & $167.4(210.6)$ & $47(44.7)$ & $54.7(47)$ & $103.2(64.1)$ & | 37.7 ( I | 8.4) \\
\hline
\end{tabular}

(Numbers in parentheses are standard deviation. Opioid doses are mg intravenous morphine. $\left({ }^{*}\right) \mathrm{p}<0.05$ and $\left({ }^{\wedge}\right) \mathrm{p}<0.0 \mathrm{I}$ by paired $\mathrm{t}-$ test.)

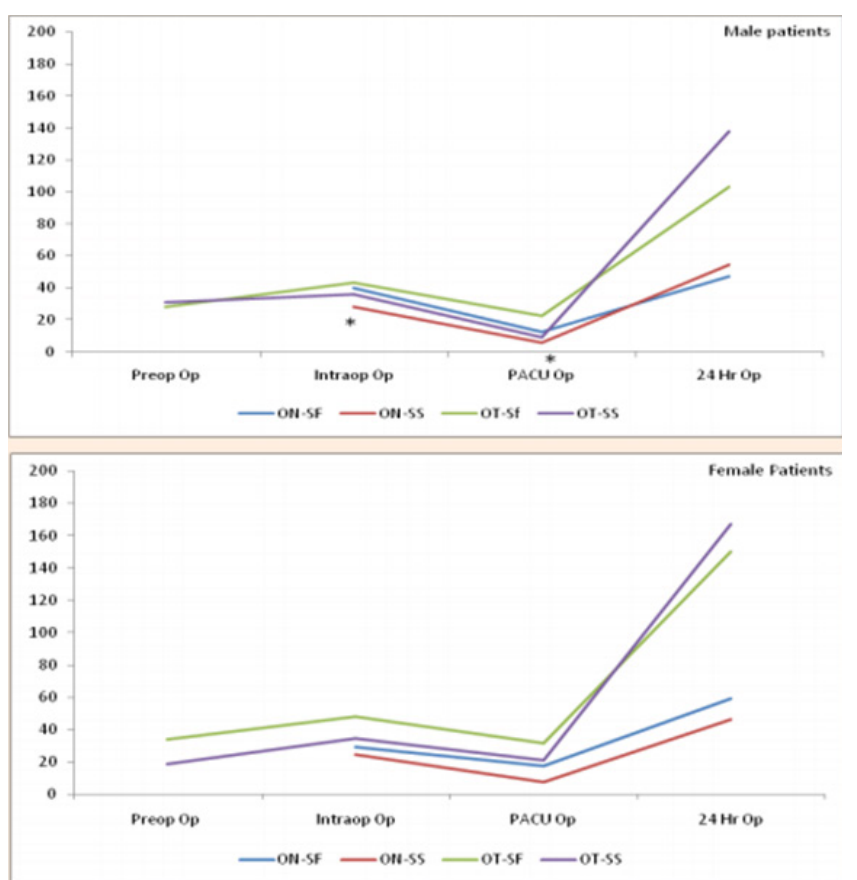

Figure I Perioperative opioid doses for opioid naïve $(\mathrm{ON})$ and opioid tolerant (OT) patients that were administered combined sciatic-femoral (SF) or sciatic-saphenous (SS) nerve blocks, expressed in mg intravenous Morphine. (*) $\mathrm{p}<0.05$ by Student's paired t-test.

Combined popliteal sciatic and femoral or saphenous nerve blockade for foot and ankle surgery either as an anesthetic technique in lieu of general or neuraxial anesthesia or as an opioid sparing intraoperative and postoperative analgesic technique has been advocated as a preferred modality for perioperative pain control. ${ }^{14,15}$ Acomparison between the techniques for blockade of the saphenous nerve demonstrated that the trans-sartorial approach in the thigh provides superior results with respect to level of analgesia and the absence of quadriceps weakness that occurs with the high femoral approach. ${ }^{16}$ Ultrasound guided nerve blockade has been associated with improved pain control compared to nerve stimulator techniques and the different technology used in this study for blockade of the saphenous nerve compared to the femoral nerve could have influenced the difference in analgesia between the two groups. ${ }^{17}$

In this study the current levels that were considered acceptable for effective nerve stimulator guided nerve blockade were within the clinically effective range of less than $0.6 \mathrm{~mA}$ that is associated with successful analgesia and greater than the injury range of $0.3 \mathrm{~mA}$ that could indicate intra-neural needle tip placement. ${ }^{18,19}$ Local anesthetic injection within the paraneuracompartment proximal to the sciatic, saphenous or femoral nerve under ultrasound guidance is highly effective in achieving analgesia in comparison to other sites of injection around the nerves and was the universal needle tip localization point in this study. ${ }^{20}$ Conventional ultrasound technology allows this localization and this site poses a reduced risk of nerve injury than a subparaneuralor intraepineural injection site that requires high definition imaging processors not readily available in many centers. ${ }^{21}$

ON females in the SS group had significantly lower PACU pain scores than those in the SF group. All groups for both genders that received SS nerve blockade had reduced opioid dosage requirements for breakthrough pain in PACU and this was statistically significant for opioid naïve males. The observed improved analgesia is similar to the report of complete absence of pain in $77 \%$ of patients receiving saphenous nerve blockade for lower extremity surgery. ${ }^{22}$ Combined sciatic and femoral nerve blockade has been observed to produce slightly lower analgesic efficacy compared to sciatic nerve blockade alone for lower extremity surgery however targeted blockade of the saphenous component of the femoral nerve and its influence on postoperative pain control were not evaluated. ${ }^{23}$ The lower intraoperative opioid dosages administered to opioid naïve females could account for the differences noted in the lower pain scores and immediate postoperative rescue analgesic opioid dosages for opioid naïve males. This gender based bias could have influenced the results observed in this study.

Opioid requirements and pain scores 24 hours postoperatively were close to the respective intraoperative and preoperative values for opioid naïve patients but were elevated in opioid tolerant patients as demonstrated in Figure 1. The presence of chronic pain and opioid 
tolerance causing higher postoperative analgesic requirements in patients with nerve blockade has been described and an individualized pain treatment program is recommended for this association. ${ }^{24}$

The validity of the results was tested by the one way ANOVA formula and the $\mathrm{F}$ and $\mathrm{p}$ results for the variable groups are plotted in Figure 2. Those comparison groups with higher $\mathrm{F}$ values and lower $\mathrm{p}$ values are considered statistically significant and these included the PACU pain score and PACU opioid doses for OT and ON patients. The limitation of this statistical analysis is that a high $\mathrm{F}$ score can result when sample sizes are small or there exists a wide range of values for the data points in any given group. The use of the discrete pain score as opposed to the visual analog score continuum was a limitation in this study. The pain score was employed because nursing staff were assessing the patient during the postoperative period and recording the data in the patient record for later review by the investigators.

Another limitation is the lack of standardization of the personnel administering the nerve blocks although the same regional anesthesia faculty supervisors were kept constant throughout the study period. Future studies using randomized patient selection and standardized analgesic dosages and expert regional anesthesia practitioners are needed to substantiate these data.

\section{Conclusion}

Saphenous nerve blockade combined with popliteal sciatic nerve blockade resulted in lower immediate postoperative pain scores in opioid naïve females and reduced immediate postoperative rescue opioid analgesic dosages in all patient groups. The differences in pain scores and analgesic requirements were not present 24 hours postoperatively. Elevated dosages of rescue opioid analgesic medications were required for opioid tolerant patients with effective nerve blockade 24 hours postoperatively.

\section{Conflicts of Interest}

The authors do not have any Conflict of interests.

\section{Acknowledgments}

None.

\section{Funding}

None.

\section{References}

1. Tran DQ, Clemente A, Finlayson RJ. A review of approaches and techniques for lower extremity nerve blocks. Can J Anaesth. 2007;54(11):922-934.

2. Singelyn FJ. Single injection applications for foot and ankle surgery. Best Pract Res Clin Anaesthesiol. 2002;16(2):247-254.

3. Beaulieu P, Babin D, Hemmerling T. The pharmacodynamics of ropivacaine and bupivacaine in combined sciatic and femoral nerve blocks for total knee arthroplasty. AnesthAnalg. 2006;103(3):768-774.

4. Blumenthal S, Borgeat A, Neudörfer C, et al. Additional femoral catheter in combination with popliteal catheter for analgesia after major foot and ankle surgery. Br J Anaesth. 2011;106(3):387-393.

5. Duggal S, Flics S, Cornell CN. Intra-articular analgesia and discharge to home enhance recovery following total knee replacement. HSS $J$ 2015;11(1):56-64.

6. Ishiguro S, Asano N, Yoshida K, , et al. Day zero ambulation under modified femoral nerve block after minimally invasive surgery for total knee arthroplasty: preliminary report. J Anesth. 2013;27(1):132-134.

7. Chisholm MF, Bang H, Maalouf DB, et al. Postoperative analgesia with saphenous block appears equivalent to femoral nerve block in ACL reconstruction. HSS J. 2014;10(3):245-251.

8. van der Wal M, Lang SA, Yip RW. Trans-sartorial approach for saphenous nerve block. Can J Anaesth. 1993;40(6): 542-546.

9. Benzon HT, Sharma S, Calimaran A. Comparison of the different approaches to saphenous nerve block. Anesthesiology. 2005;102(3):633638

10. Hsu LP, Oh S, Nuber GW, et al. Nerve block of the infrapatellar branch of the saphenous nerve in arthroscopy: a prospective, doubleblinded, randomized, placebo-controlled trial. J Bone Joint Surg Am. 2013;95(16):1465-1472.

11. Anns JP, Chen EW, Nirkavan N, et al. A comparison of sartorius versus quadriceps stimulation for femoral nerve block: a prospective randomized double blind controlled study. Anesth Analg. 2011;112(3):725-731.

12. Chen J, Lesser J, Hadzic A, et al. The importance of the proximal saphenous nerve block for foot and ankle surgery. Reg Anesth Pain Med. 2013;38(4):372.

13. Tumber PS. Optimizing perioperative analgesia for the complex pain patient: medical and interventional strategies. Can J Anaesth. 2014;61(2):131-140.

14. Singelyn FJ. Single-injection applications for foot and ankle surgery. Best Pract Res Clin Anaesthesiol. 2002;16(2):247-254.

15. Donohue CM, Goss LR, Metz S, et al. Combined popliteal and saphenous nerve blocks at the knee: an underused alternative to general or spinal anesthesia for foot and ankle surgery. J Am Podiatr Med Assoc. 2004;94(4):368-374.

16. Benzon HT, Sharma S, Calimaran A. Comparison of the different approaches to saphenous nerve block. Anesthesiology. 2005;102(3):633638

17. Perlas A, Brull R, Chan VW, et al. Ultrasound guidance improves the success of sciatic nerve block at the popliteal fossa.RegAnesth Pain Med. 2008;33(3):259-265.

18. Aguirre J, Valentin Neudörfer C,Ekatodramis G, et al. Ultrasound guidance for sciatic nerve block at the popliteal fossa should be compared with the best motor response and the lowest current clinically used in neurostimulation technique. Reg Anesth Pain Med. 2009;34(2):182-183.

19. Robards C, Hadzic A, Somasundaram L, et al. Intraneural injection with low-current stimulation during popliteal sciatic nerve block. Anesthesia \& Analgesia. 2009;109(2):673-637.

20. Tsai PB, Karnwal A, Kakazu C, et al. Efficacy of an ultrasound-guided subsartorial approach to saphenous nerve block: a case series. Can $J$ Anaesth. 2010;57 (7):683-638.

21. Perlas A, Wong P, Abdallah F, et al. Ultrasound-guided popliteal block through a common paraneural sheath versus conventional injection: A prospective, randomized, double-blind study. Reg Anesth and Pain Med. 2013;38(3):218-225.

22. Karmakar MK, Shariat AN, Pangthipampai P, et al. High-definition ultrasound imaging defines the paraneural sheath and the fascial compartments surrounding the sciatic nerve at the popliteal fossa. RegAnesth Pain Med. 2013;38(5):444-451.

23. Lee KT, Park YU, Jegal H, et al. Femoral and sciatic nerve block for hindfoot and ankle surgery. J Orthop Sci. 2014;19(4):546-551.

24. Souzdalnitski D. Regional anesthesia and co-existing chronic pain.Curr Opin Anaesthesiol. 2010;23(5):662-670. 